\title{
Optimized lymph node dissection range during progression of lower thoracic esophageal squamous cell carcinoma in the latest therapeutic surgical strategy: A retrospective analysis
}

\author{
HIROKI HARADA ${ }^{1}$, KEI HOSODA ${ }^{1}$, HIROMITSU MORIYA ${ }^{1}$, HIROAKI MIENO ${ }^{1}$, AKIRA EMA $^{1}$, \\ MARIE WASHIO $^{1}$, YOSHIMASA KOSAKA ${ }^{1,2}$, MASAHIKO WATANABE ${ }^{1}$ and KEISHI YAMASHITA ${ }^{1,3}$ \\ Departments of ${ }^{1}$ Surgery and ${ }^{2}$ Breast and Endocrine Surgery, Kitasato University School of Medicine; \\ ${ }^{3}$ Division of Advanced Surgical Oncology, Department of Research and Development Center for New Medical Frontiers, \\ Kitasato University School of Medicine, Sagamihara, Kanagawa 252-0375, Japan
}

Received October 31, 2017; Accepted June 6, 2018

DOI: $10.3892 / \mathrm{ol} .2018 .9030$

\begin{abstract}
The distribution of lymph node metastases, including recurrences, remains elusive in lower thoracic esophageal squamous cell carcinoma (LtESCC). The present study was a retrospective investigation into the optimized lymph node dissection range during LtESCC. Esophagectomies were performed on 163 patients with ESCC between 2009 and 2016, among whom 41 patients with LtESCC were examined. The rates of pathological and potential (including recurrences) metastases to lymph nodes and the prognosis (median, 34 months) were determined. Preoperative Docetaxel, Cisplatin and 5-fluorouracil chemotherapy was administered in $>60 \%$ of cStage II/III LtESCC. During stage progression, abdominal lymph node metastasis rapidly becomes aggressive in LtESCC and lymph node metastases to the para-aortic area were more dominant than cervical and recurrent laryngeal nerve (RLN) areas. There were few control failures of regional lymph node metastases in LtESCC with surgery, if 1 unique case with cStage III who had metastases and recurrences of multiple lymph nodes during the clinical course was excluded. Defective lymph node dissection around the RLN did not worsen LtESCC prognosis with no RLN palsy. In the context of the potent preoperative chemotherapy and esophagectomy, lymph node dissection of cervical, para-aortic and RLN areas are putatively not mandatory to all LtESCC patients.
\end{abstract}

Correspondence to: Dr Keishi Yamashita, Department of Surgery, Kitasato University School of Medicine, 1-15-1 Kitasato, Minami-ku, Sagamihara, Kanagawa 252-0375, Japan

E-mail: keishi23@med.kitasato-u.ac.jp

Key words: esophageal squamous cell carcinoma, lower thoracic esophageal carcinoma, recurrent laryngeal nerve, lymph node metastasis, Docetaxel, Cisplatin and 5-fluorouracil

\section{Introduction}

In Japan, esophageal squamous cell carcinoma (ESCC) is located in the middle thoracic esophagus (Mt) region (51.6\%), lower thoracic esophagus (Lt) region (24.2\%), upper thoracic esophagus (Ut) region (13.4\%), abdominal thoracic esophagus (Ae) region (4.5\%), and cervical esophagus $(\mathrm{Ce})$ region $(4.0 \%)$ (1). There have been only a few reports of the distribution of lymph node metastases in cases of lower thoracic ESCC (LtESCC).

LtESCC leads to significantly higher lymph node metastases to the abdomen than to any other location (2). A previous study reported that three-field lymph node dissection improved 5-year overall survival (OS) rates in LtESCC patients (3). However, another study questioned whether three-field lymph node dissection contributed to better OS in whole LtESCC patients (4). In LtESCC patients, metastases to para-aortic lymph nodes are also observed in $25.5 \%$ of cases. Therefore, para-aortic lymph node dissection should have been considered in cases of positive perigastric lymph node metastases (5). Furthermore, poor patient prognosis has been observed in LtESCC patients with positive recurrent laryngeal nerve (RLN) lymph node metastases (6).

We have been committed to developing novel potent chemotherapeutic regimens to control early treatment failures in clinical stage (cStage) II/III ESCC by adding Docetaxel (DTX) to Cisplatin (CDDP) and 5-fluorouracil (together referred to as DCF). In our hospital, DCF neoadjuvant chemotherapy (NAC) had a better prognosis than CF NAC $(7,8)$. Describing the status of lymph node metastases or recurrence with the latest treatment strategy will be useful for developing future therapeutic strategies.

In this study, patients with LtESCC were assessed with regard to the rates of lymph node metastases allowing for recurrence in the context of the latest multimodality treatments, as well as their influence on patient prognosis. These data could be important for determining the optimal range of lymph node dissection in LtESCC. 


\section{Materials and methods}

Patients. A prospective database of 464 patients with thoracic ESCC, provided by the esophageal Cancer Board of Kitasato University of Medicine, Departments of Gastroenterology, Surgery, and Radiology (Sagamihara, Japan), between January 1, 2009 and March 31, 2016, was analyzed. Among 163 patients who had undergone surgery at Kitasato University Hospital (Sagamihara, Japan), 41 patients diagnosed with LtESCC, excluding two cases of cStage IV cancer, were included in the study. The median follow-up was 34.3 months (range, 2.3-87.4 months).

Staging. The tumor stage was classified according to the 6th edition of the Union for International Cancer Control TNM (UICC TNM) for esophageal cancer. The definition of positive lymph node metastasis was a short diameter of $1 \mathrm{~cm}$ on CT. Until 2010, cases were diagnosed as positive by a radiologist, but after 2011, a short diameter of $1 \mathrm{~cm}$ was defined as positive. We examined the patients limited to surgery alone $(n=62)$ with regard to diagnostic accuracy, after we ruled out those with NAC to allow for downstage of the disease. As a result, the diagnosis rate of preoperative lymph node metastasis was proved to be sensitivity of $100 \%$ (8/8) and specificity of $68.5 \%$ (37/54), if we used the cut-off size of lymph node swelling with $1 \mathrm{~cm}$.

NAC Treatments in cStage II/III ESCC. Patients received 3 cycles of DCF NAC every 3 weeks. The regimen consisted of DTX $70-75 \mathrm{mg} / \mathrm{m}^{2}$ given as a 1 -h intravenous infusion on day 1 , CDDP $70-75 \mathrm{mg} / \mathrm{m}^{2}$ as a $2-\mathrm{h}$ intravenous infusion on day 1 , and $5-\mathrm{FU} 750 \mathrm{mg} / \mathrm{m}^{2}$ as a continuous $24-\mathrm{h}$ peripheral infusion on days 1-5. From February 2011, the starting doses of DTX and CDDP were increased to $75 \mathrm{mg} / \mathrm{m}^{2} \mathrm{CF}$ NAC with CDDP plus 5-FU was repeated twice every 3 weeks.

In patients whose response to the first or second course of chemotherapy was progressive disease or who could not tolerate the side effects of DCF or CF NAC, the next course of chemotherapy was not given. Non-responders with stable disease received scheduled cycles of NAC.

Surgical Treatment. A standard esophagectomy was performed according to the McKeown method (i.e., right thoracotomy followed by laparotomy and neck incision with cervical anastomosis), and three-field (thoracoabdominal and cervical) lymph node dissection was also performed if indicated. After surgery, the lymph nodes were separated from the resected esophagus and adjacent tissue, and they were assigned specific numbers indicating the location of the lymph nodes, in accordance with the guideline of the Japanese Society for Esophageal Disease (JSED) (9). R0, R1, and R2 surgical resections were defined as microscopically and curatively resected, microscopically remnant, and macroscopically remnant cancer cells, respectively.

Statistical analyses. All statistical analyses were performed using JMP ${ }^{\circledR} 11$ software (SAS Institute, Inc., Cary, NC, USA). Frequency tables were analyzed using the $\chi^{2}$ test, with the likelihood ratio or Fisher's exact test used to determine the significance of differences between categorical variables. OS was measured from the date of death or censored at the date of the last follow-up evaluation. Survival was estimated by a life table using the Kaplan-Meier method and compared by the log-rank test. Differences between results of comparative tests were considered significant if the two-sided P-value was less than 0.05 .

\section{Results}

Prognosis of LtESCC who underwent surgery with curative intent. Among the 464 thoracic esophageal cancer, esophagectomy was recommended to be performed with curative intent in 163 cases $(35.1 \%)$ of the thoracic ESCC cases; the remaining 301 cases $(64.9 \%)$ did not have surgical treatment. Salvage surgery after curative definitive chemoradiotherapy (dCRT) was not considered surgical treatment. The 5-year OS rate was 75.2 and $33.7 \%$ for the surgical and non-surgical treatment groups, respectively, and this difference was significant $(\mathrm{P}<0.0001$; Fig. 1A). Cancer of the Ut, Mt, and Lt was found in 19, 101, and 43 cases, respectively, and 5-year OS rates for these patients were $81.9,72.3$, and $78.1 \%$, respectively. There was no significant difference in prognosis among these groups $(\mathrm{P}=0.7887$; Fig. 1B).

Prognosis of Lt ESCC with esophagectomy and lymph node dissection. When the data were stratified by clinical stage for patients with LtESCC, the 5-year OS rates were 100\% in cStage I patients, $84.6 \%$ in cStage II patients, and $60.9 \%$ in cStage III patients. While there was no significant difference in prognosis among these patients $(\mathrm{P}=0.1525)$, OS decreased with higher cStage (Fig. 2A).

The characteristics of the 41 patients with LtESCC, with cStage I to cStage III, are presented in Table I. One patient in cStage III had inoperable cancer. Video-Assisted Thoracic Surgery (VATS) esophagectomy was performed more often in the earlier stage than in the more advanced stage $(\mathrm{P}=0.0007)$. Regarding the range for lymph node dissection, no significant differences were observed between two-field and three-field dissection methods $(\mathrm{P}=0.4294)$. DCF NAC was performed in 66.7 and $60.0 \%$ of patients with cStages II and III LtESCC, respectively. Patients with cStage I LtESCC who underwent CRT (S1+Radiation) prior to surgery had concomitant advanced hypopharyngeal carcinoma (Table I).

Lymph node dissection rates are shown in Fig. 2B. In cervical lymph nodes representing yellow color, No. 101 was dissected in more than $80 \%$ of patients, but No. 104 was dissected in $30.8 \%$ of patients. In upper mediastinal lymph nodes representing green and light blue colors, Nos. 105 and 106recR lymph nodes along the right RLN (green) were dissected in 94.9 and $84.6 \%$ of patients, respectively, and lymph nodes along the left RLN lymph nodes (Nos. 106recL and 106tbL) (light blue) were dissected in 82.1 and $71.8 \%$ of patients, respectively. In middle mediastinal lymph node representing dark blue colors, No. 109 was dissected in only $46 \%$ of patients, whereas Nos. 107 and 108 were dissected in 87 and $92 \%$ of patients, respectively. This is putatively because No. 109 was often removed with No. 107. In the lower mediastinum representing purple color, No. 110 was dissected in $92 \%$ of patients, but Nos. 111 and 112 were dissected in only 54 and $28 \%$ of patients, respectively. This is also because Nos. 111 

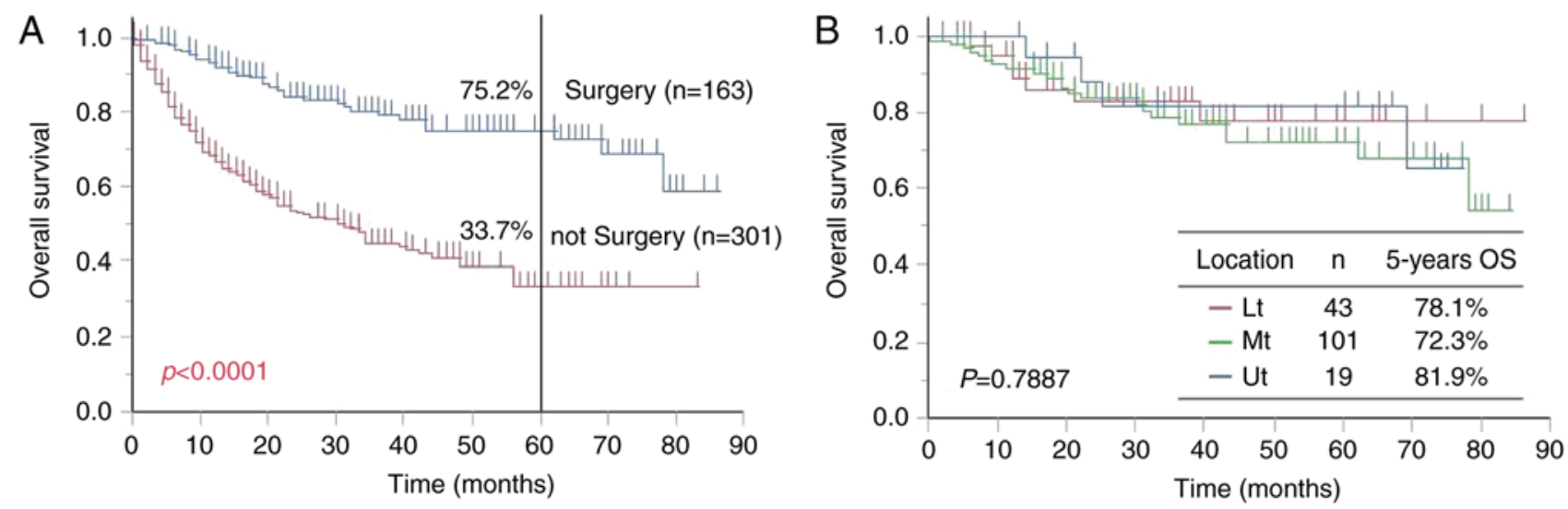

Figure 1. Five-year overall survival rates (A) for patients who underwent surgery and those who did not undergo surgery for thoracic esophageal squamous cell carcinoma and for (B) tumor locations in the Lt, Mt, and Ut regions. Lt, lower thoracic; Mt, middle thoracic; Ut, upper thoracic.
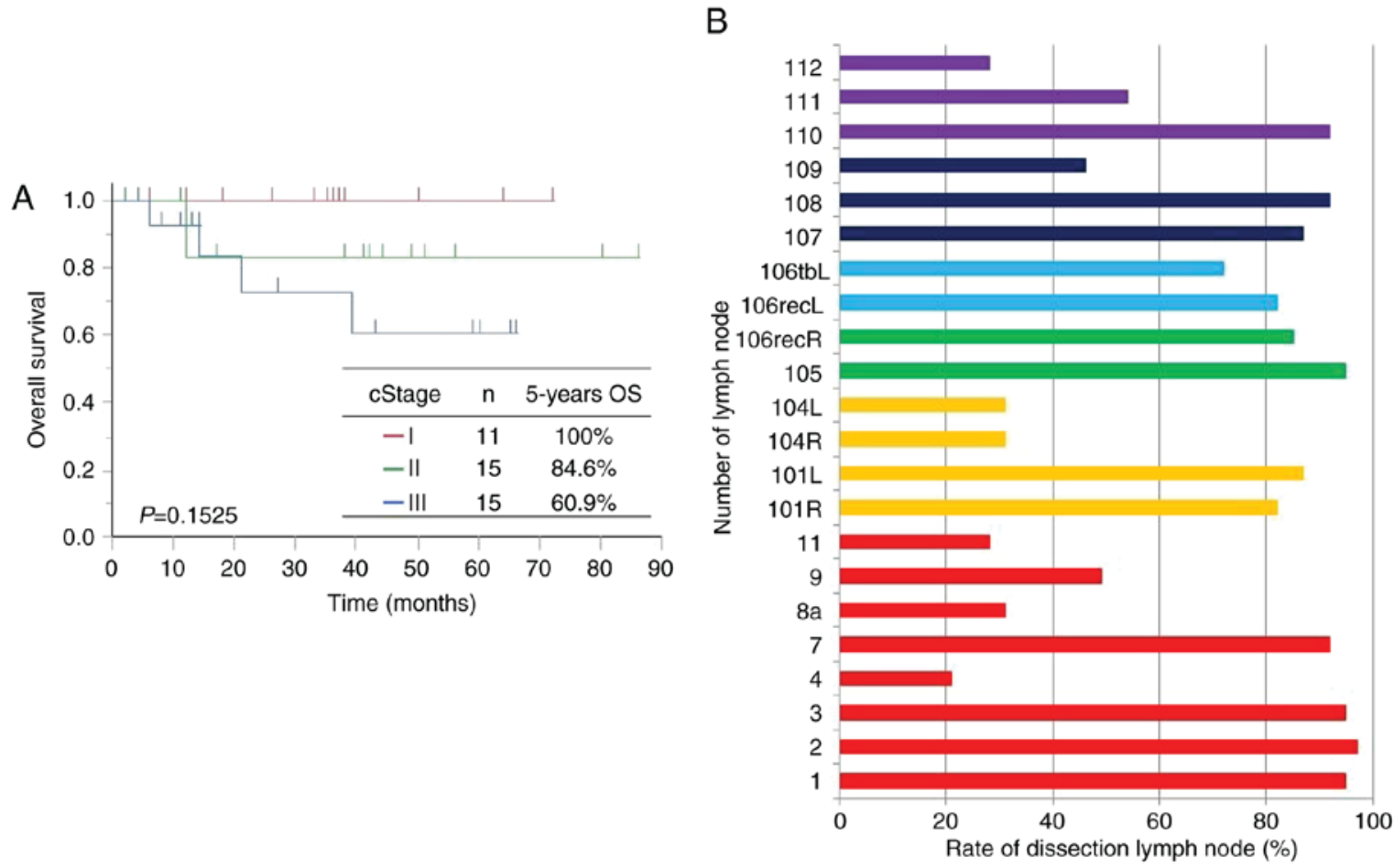

Figure 2. (A) Five-year overall survival rates of patients with lower thoracic esophageal squamous cell carcinoma in cStages I, II, and III. (B) Frequency of lymph node dissection at Kitasato University Hospital. Red, abdominal lymph node; yellow, cervical lymph node; green, right upper mediastinal lymph node; light blue, left upper mediastinal lymph node; dark blue, middle mediastinal lymph node; purple, lower mediastinal lymph node.

and 112 were often removed with No. 110. In the abdomen representing red color, lymph nodes were dissected around the cardia, the small curvature, and the celiac artery in about $90 \%$ of patients. However, lymph nodes Nos. 4, 8a, 9, and 11 were dissected in $20.5,30.8,48.7$, and $28.2 \%$ of patients, respectively. No. 9 may be dissected with No. 7 .

Distribution of pathological lymph node metastasis and metastatic rates in LtESCC with esophagectomy according to $c$ Stage. The rate of metastasis for each lymph node, according to cStage, is shown in Fig. 3. In cStage I patients, the rates of metastases to lymph node Nos. 106recR, 108, and 110 were 9.1, 9.1, and $9.1 \%$, respectively, with no lymph node metastases observed to the abdominal cavity (Fig. 3A). In cStage II patients, the rate of metastases to cervical lymph node No. 101 was $6.7 \%$, whereas the rates of metastases to thoracic lymph node Nos. 106recL and 108 were 13.3 and $13.3 \%$, respectively. On the other hand, the rates of metastases to abdominal lymph node Nos. 1, 2, 3, 7, and 11p were 20.1, 26.8, 6.7, 6.7, and 6.7\%, respectively (Fig. 3B). In cStage III patients, rates of metastases to lymph node Nos. 101, 106recR, 105, 106recL, and 106tbL were 7.1,7.1, 7.1, 14.3, and $0 \%$, respectively, whereas rates for lymph node Nos. 108, 110, 111, and 112 were 14.3, 7.1, 7.1, and $0 \%$, respectively. High rates of lymph node metastases were observed to abdominal lymph node Nos. 1, 2, 3, 7, 8 a, 9, and $11 \mathrm{p}(35.7,21.4,35.7,28.6,7.1,14.3$, and 7.1\%, respectively; Fig. 3C). These results suggest that abdominal lymph node metastases may increase rapidly with progression. 
Table I. Patients' characteristics.

\begin{tabular}{|c|c|c|c|c|c|}
\hline Variable & Number $(n=41)$ & cStage I $(n=11)$ & cStage II $(n=15)$ & cStage III $(n=15)^{\mathrm{a}}$ & P-value \\
\hline Age (years) & & & & & 0.9049 \\
\hline$<69$ & 21 & 6 & 7 & 8 & \\
\hline$\geq 69$ & 20 & 5 & 8 & 7 & \\
\hline Sex & & & & & 0.0793 \\
\hline Male & 34 & 11 & 12 & 11 & \\
\hline Female & 7 & 0 & 3 & 4 & \\
\hline pT factor & & & & & 0.0030 \\
\hline pT0 & 4 & 0 & 2 & 2 & \\
\hline pT1 & 15 & 10 & 5 & 0 & \\
\hline pT2 & 4 & 0 & 2 & 2 & \\
\hline pT3 & 16 & 1 & 6 & 9 & \\
\hline pT4 & 1 & 0 & 0 & 1 & \\
\hline Lymph vessels invasion & & & & & 0.1261 \\
\hline Presence & 18 & 5 & 4 & 9 & \\
\hline Absence & 22 & 6 & 11 & 5 & \\
\hline Blood vessels invasion & & & & & 0.0686 \\
\hline Presence & 19 & 3 & 6 & 10 & \\
\hline Absence & 21 & 8 & 9 & 4 & \\
\hline Pathological stage & & & & & 0.0487 \\
\hline I & 12 & 8 & 4 & 0 & \\
\hline IIA & 6 & 0 & 2 & 4 & \\
\hline IIB & 5 & 2 & 2 & 1 & \\
\hline III & 11 & 1 & 5 & 5 & \\
\hline IVA & 1 & 0 & 0 & 1 & \\
\hline IVB & 1 & 0 & 0 & 1 & \\
\hline $\mathrm{CR}$ & 4 & 0 & 2 & 2 & \\
\hline Procedure of esophagectomy & & & & & 0.0007 \\
\hline Open & 14 & 0 & 3 & 11 & \\
\hline VATS & 26 & 10 & 12 & 4 & \\
\hline Transhiatal approach & 1 & 1 & 0 & 0 & \\
\hline Field of lymph node dissection & & & & & 0.4294 \\
\hline Two & 25 & 7 & 11 & 7 & \\
\hline Three & 15 & 4 & 4 & 7 & \\
\hline Preoperative therapy & & & & & 0.0002 \\
\hline Surgery alone & 16 & 10 & 1 & 5 & \\
\hline Cisplatin+5-FU & 5 & 0 & 4 & 1 & \\
\hline Docetaxel+Cisplatin+5-FU & 19 & 0 & 10 & 9 & \\
\hline Chemoradiotherapy & 1 & 1 & 0 & 0 & \\
\hline Reccurence of lymph node & & & & & 0.0867 \\
\hline Presence & 5 & 0 & 1 & 4 & \\
\hline Absence & 36 & 11 & 14 & 11 & \\
\hline
\end{tabular}

${ }^{\mathrm{a}}$ One patient had inoperable cancer. cStage, clinical stage; pT, Pathological T; CR, complete response; 5-FU, 5-fluorouracil.

Distribution of potential lymph node metastasis and metastatic rates in LtESCC with esophagectomy according to cStage. Then, lymph node recurrence in combination with pathological lymph node metastasis was defined as potential lymph node metastasis, and the potential lymph node metastases are shown Fig. 4. Red circles represent recurrent lymph nodes, and the rate of the metastasis was calculated after adding to pathological lymph node metastasis. This figure therefore includes metastases that had not been removed by surgery.

There were no cases of recurrent lymph node metastases in cStage I patients (Fig. 4A). Recurrence of metastases to lymph 
A
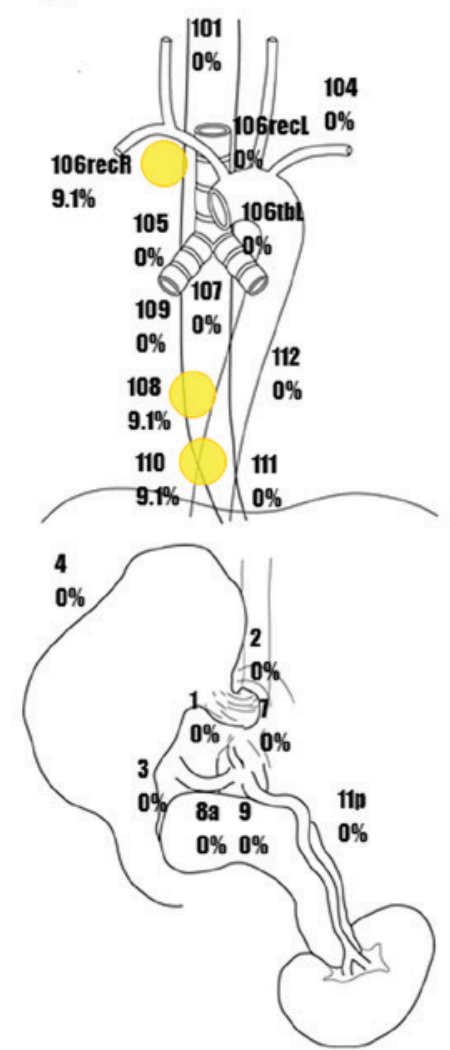

B
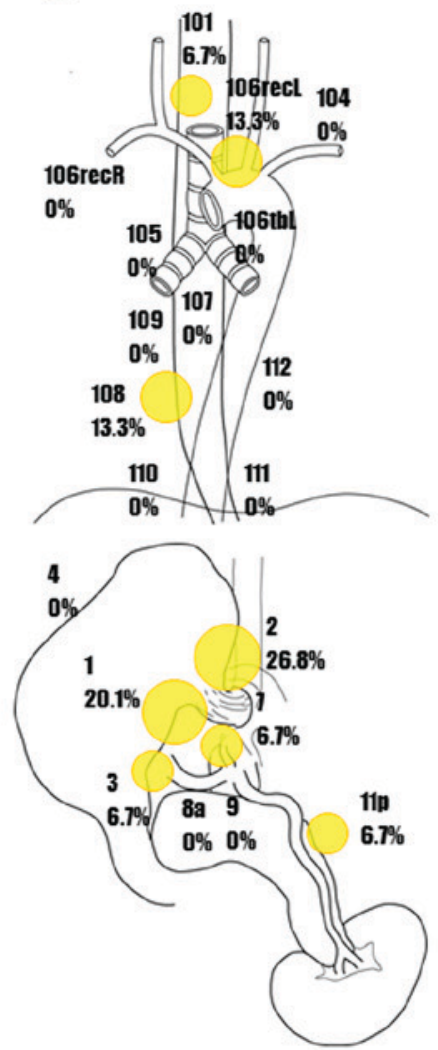

C
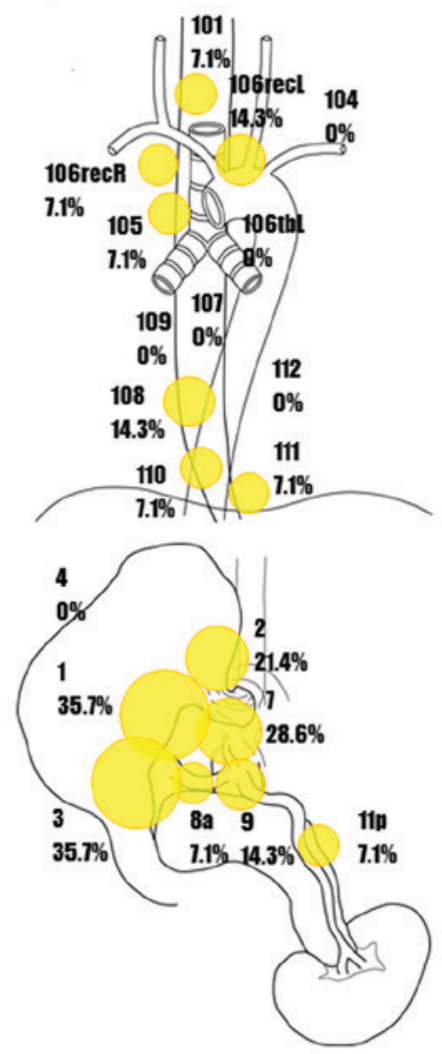

Figure 3. Rate of lymph node metastases (yellow) by cStage in patients with lower thoracic esophageal squamous cell carcinoma. (A) cStage I, (B) cStage II, and (C) cStage III. Yellow spheres represent the pathological lymph node metastases positive rate after esophagectomy. The size of the ball represents an increase in the number rate.

node Nos. 112 and 16 was added to the pathological lymph node metastases in cStage II patients (Fig. 4B). In cStage III patients, recurrence of metastases to lymph node Nos. 104, 109,110 , and 112 was observed in the cervical and thoracic areas. There was also recurrence of metastases to lymph node Nos. 7, 8a, 9, 11p, and 16 in the abdominal cavity (Fig. 4C).

Distribution of potential lymph node metastasis and metastatic rates in LtESCC with esophagectomy according to cStage. However, one case with cStage III uniquely had robust lymph node metastases (Fig. 5A), which resulted in early lymph node recurrence after surgery; this patient died due to rapid exacerbation of the disease. In this patient, recurrence of metastases shortly occurred to lymph node Nos. 104, 110, 112, 7, 8a, 9, 11p, and 16 (Fig. 5B). Since this case is considered a unique entity in cStage III LtESCC, it was excluded from the presentation of the sites of recurrent lymph node metastases of cStage III LtESCC in Fig. 5C. As a result, surgery was proved well to control recurrence of abdominal lymph node metastases even in cStage III patients. In the thoracic cavity, recurrence of metastases was confirmed to lymph node No. 109, and recurrence was only observed in abdominal lymph node No. 16 (extra-regional lymph node). In LtESCC patients, abdominal lymph nodes had a high rate of metastases, but abdominal lymph nodes were successfully dissected.

Lymph node dissection along right and left RLN and prognosis in LtESCC. Finally, the prognostic relevance of lymph node dissection around the RLN was examined. The 5-year OS rates for cases undergoing RLN dissection of lymph node Nos. 106recR $(n=6)$ and 106recL $(n=7)$ were 100 and 100\%, respectively, while those for cases not undergoing dissection were 77.7 and $77.7 \%$, respectively $(\mathrm{P}=0.3410$; Fig. $6 \mathrm{~A}$ and $\mathrm{B})$. The 5 -year OS rates of the cases in which No. 106tbL was excised $(n=29)$ and not excised $(n=12)$ were 100 and $74.3 \%$, respectively ( $\mathrm{P}=0.1392$; Fig. 6C). In LtESCC patients, prognosis was not improved by dissection of lymph nodes around the RLN.

Curative treatment after recurrence in cases with lymph node metastasis. Among the $41 \mathrm{LtESCC}$ cases, recurrences of lymph node alone were found only in 3 cases, and definitive CRT was performed in 2 among the three. Both cases survived with no relapse. In the remaining one case, chemotherapy of Nedaplatin and 5-FU was performed and the patient survived with no relapse.

\section{Discussion}

There have been few reports describing distribution of lymph node metastases including disease recurrences and the optimal dissection range of lymph nodes in LtESCC. In this current study, we have opinions with regard to efficacy of lymph node dissection for i) cervical lymph nodes; ii) para-aortic lymph nodes; and iii) lymph nodes along the RLN.

The have been clinical question with regard to necessity of cervical lymph node dissection in Lt ESCC. One of 
A
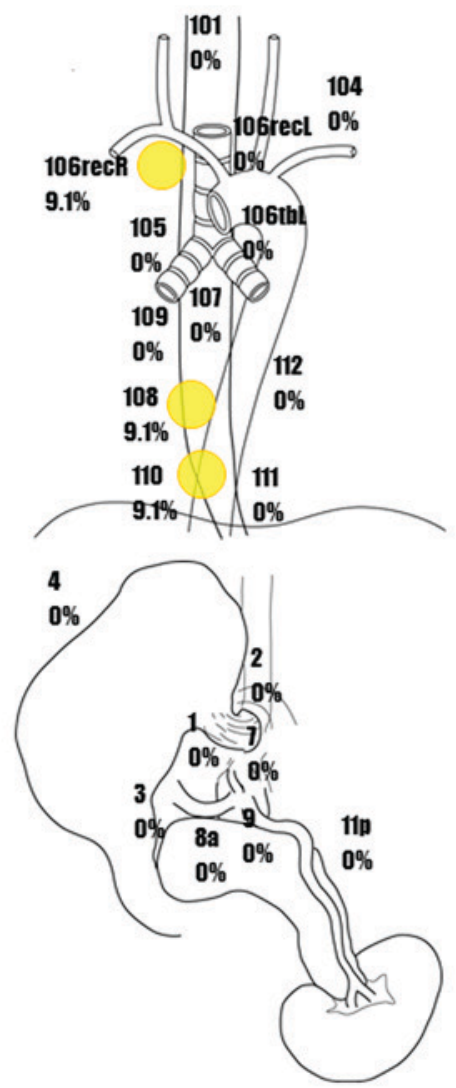

B
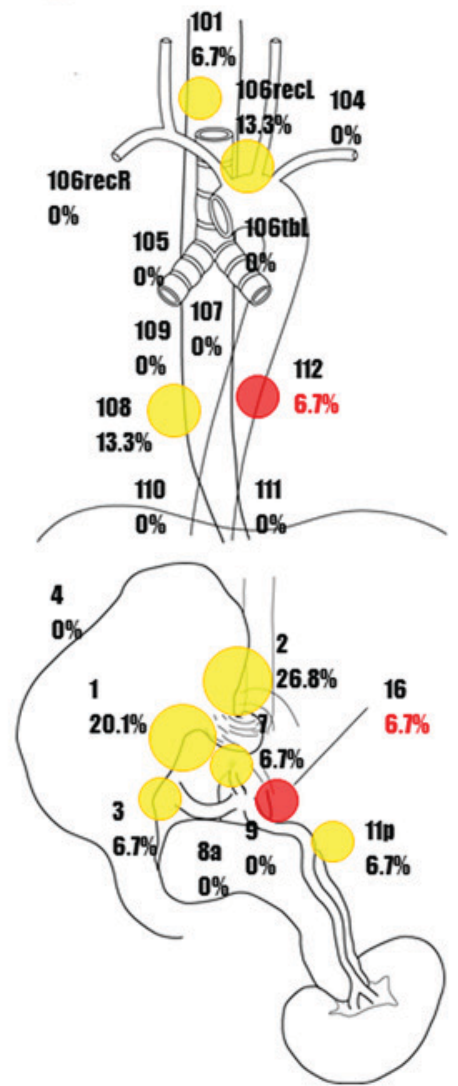

$\mathrm{C}$

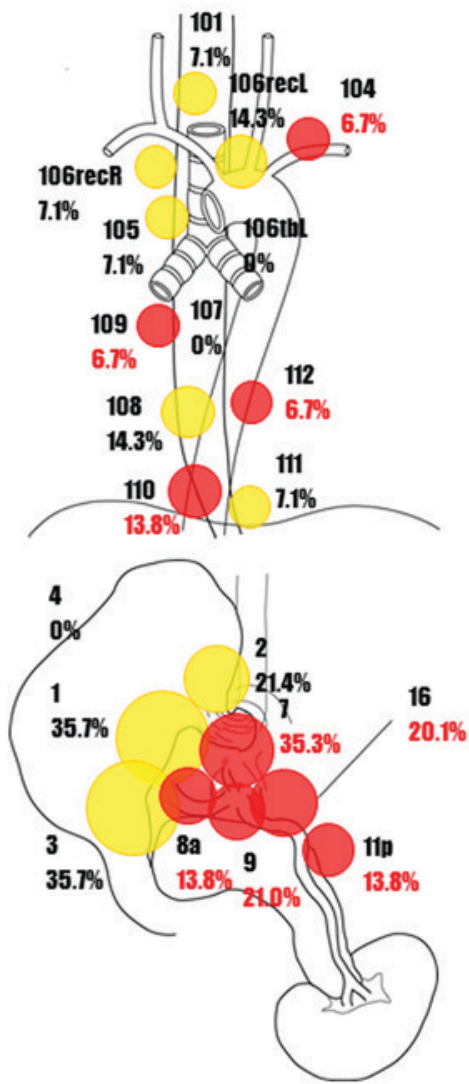

Figure 4. Rates of lymph node metastases (yellow) and lymph node recurrence (red) by cStage in patients with lower thoracic esophageal squamous cell carcinoma. (A) cStage I, (B) cStage II and (C) cStage III. Yellow spheres represent the pathological lymph node metastases positive rate after esophagectomy. Red spheres represents the recurrence rate of lymph node metastases after esophagectomy. The size of the ball represents an increase in the number rate.

the important literature in terms of this point is a report by Fujita $e t$ al that rates of metastases to cervical lymph nodes were infrequent (Nos. 101R, 101L, 104R, and 104L were $3,1,3$, and $1 \%$, respectively), which were even fewer than those with para-aortic ones (4). This incidence of metastatic cervical lymph nodes is consistent with our current study. Nishimaki et al also reported that cervical lymph node metastases in LtESCC are infrequent and do not affect prognosis. Therefore, radical esophagectomy for LtESCC patients should only be performed, limited to mediastinal and abdominal lymphadenectomy (10). On the other hand, Igaki et al critically reported that 3-field lymph node dissection in LtESCC with metastases to the upper and middle mediastinal lymph nodes results in better 5-year OS (30.0\%) than the use of two-field dissection (5.6\%; $\mathrm{P}=0.005)$, suggesting that cervical dissection might play a given role in improving prognosis in the very limited LtESCC (3). Nevertheless, Igaki et al also reported that 3-field lymph node dissection did not improve survival in LtESCC with no metastases to mediastinal lymph nodes (3). Allowing for these data, cervical lymph node dissection may be necessary in the very limited LtESCC, eg. cases with clinically lymph node metastases in the upper and/or middle mediastinum.

In this current study, we experienced 1 unique stage III LtESCC with cervical lymph node recurrence, however this case is very unique in that there were robust metastases and recurrences to multiple lymph nodes during the clinical curse. In addition, there was a report describing that patient prognosis is better in cervical lymph node recurrences to the neck (11). In cases of recurrent cervical lymph node metastases alone, salvage surgery can be performed, or even combined with CRT.

Rates of metastases were as high as $36,26,29$, and $32 \%$ to abdominal lymph node Nos. 1, 2, 3, and 7 and more frequent than those in cervical and mediastinal lymph nodes (4), which are again consistent with other's (2) and our current study. Importantly, in this study, regional lymph nodes could be well controlled, and recurrences were few.

Unique case described in the cervical lymph nodes only showed recurrence of the regional lymph nodes in the abdomen. Except this unique 1 case, para-aortic lymph node metastases are concerns in LtESCC. Shimada et al reported that para-aortic lymph node metastases or recurrence were recognized in $25.5 \%$ of LtESCC (5). In our hospital, recurrence rates in para-aortic lymph nodes was relatively high: 6.7 and $20.1 \%$ of LtESCC patients with cStages II and III, respectively. However, the recurrences of the para-aortic lymph nodes in our study were diagnosed after surgery, and it was not seen before surgery. Therefore, it is difficult to apply the NAC to such cases before surgery. As it has been reported that recurrence of para-aortic lymph nodes after surgery may be cured by performing definitive CRT after recurrence (12), preventive lymph node dissection is considered to be overtreatment for the para-aortic lymph nodes. 
A Particular case
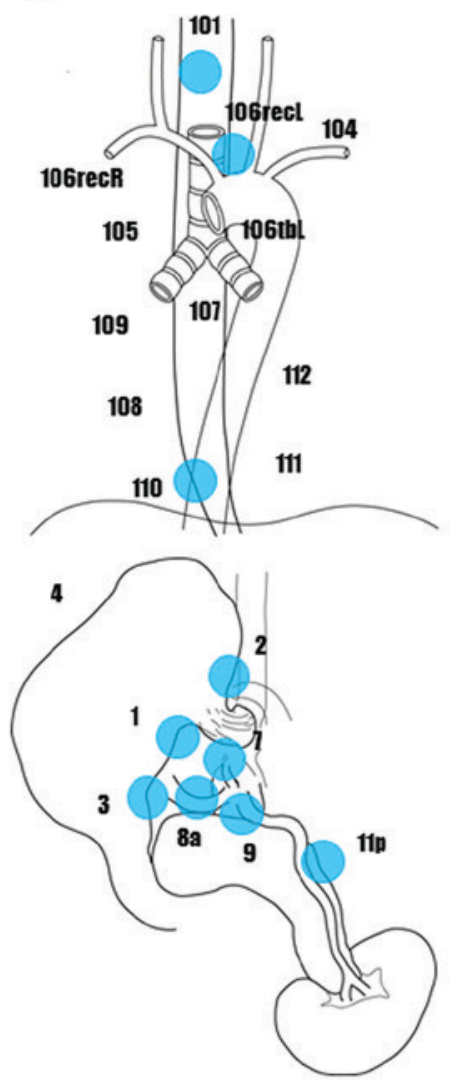

B Particular case (recurrence)
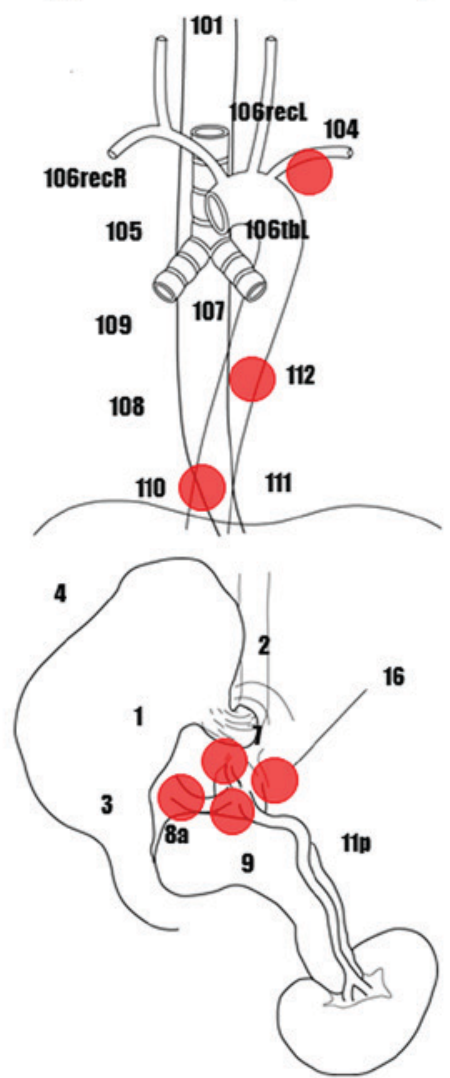

C Excluding particular case

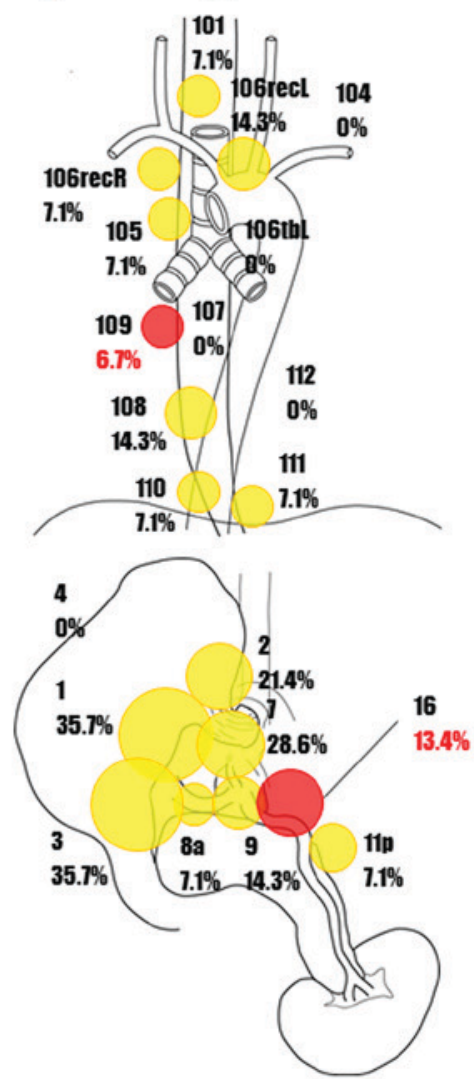

Figure 5. Rates of lymph node metastases and lymph node recurrence in patients with lower thoracic esophageal squamous cell carcinoma in cStage III. (A and B) A particular case in which frequent positive lymph node metastases were observed after surgery; this patient suffered mortality due to rapid exacerbation of the disease. The blue and red spheres represent the pathological lymph node metastases positive and the recurrence of lymph node metastases positive after esophagectomy. (C) Rates of lymph node metastases (yellow) and lymph node recurrence (red) excluding the patient described in A and B. The yellow and red spheres represent the pathological lymph node metastases and the recurrence rate of lymph node metastases after esophagectomy excluding particular case. The size of the ball represents an increase in the number rate.
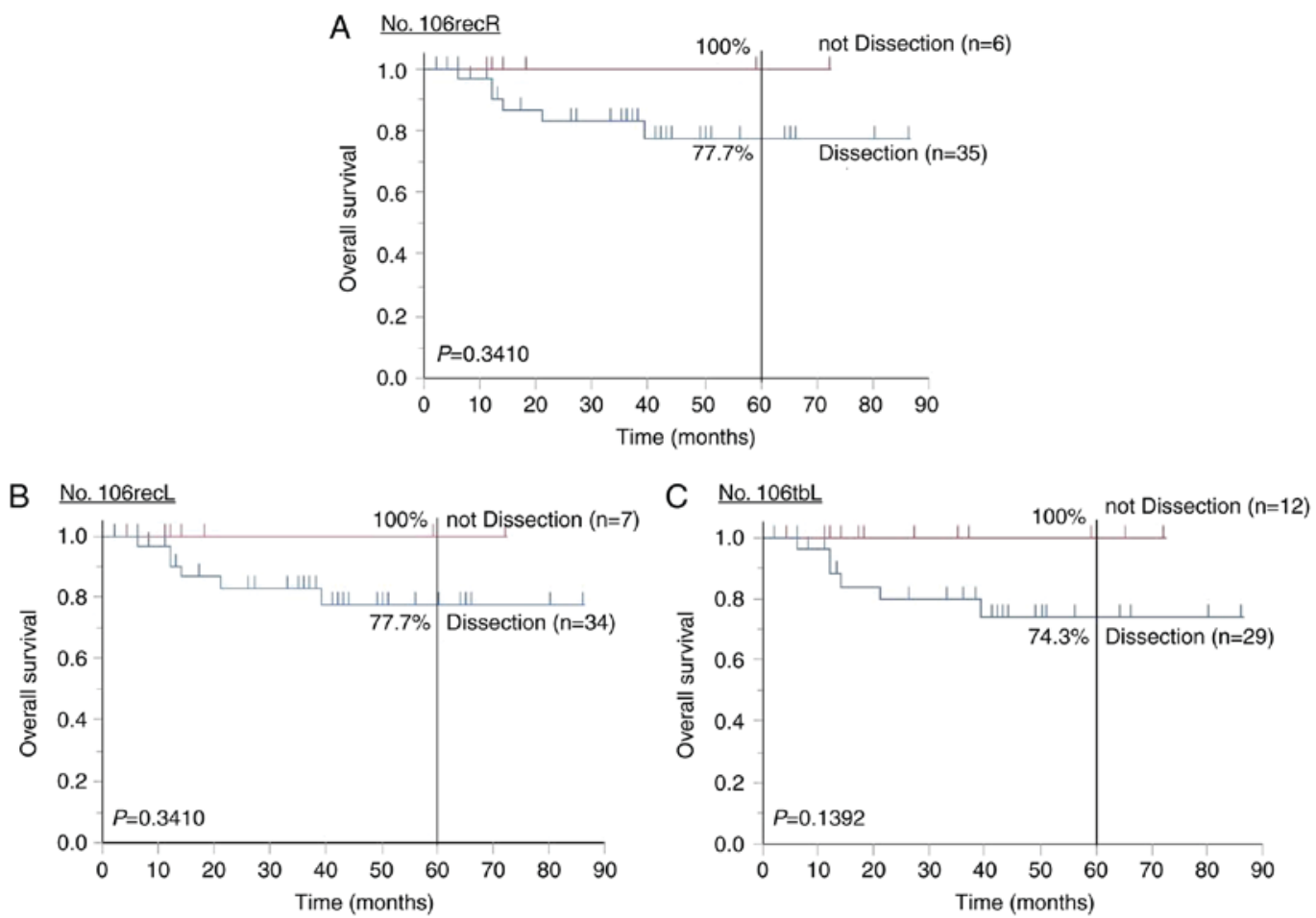

Figure 6. Five-year overall survival rates for patients with and without dissection of lymph node numbers (A) 106recR, (B) 106recL and (C) 106tbL for lower thoracic esophageal squamous cell carcinoma. 
Lymph node dissection along RLN is another debate issue in LtESCC. The rates of metastases to lymph nodes Nos. 106recR, 106recL, and 106tbL were 11, 11, and 3\%, respectively, which were further infrequent than those with metastatic para-aortic lymph nodes (4). This report result is re-capitulated in Ye's one, where the 3-year survival rate was $29.3 \%$, and prognosis was poor in patients with metastases to RLN lymph nodes (6). In cases of RLN lymph node metastases in our hospital, rates of metastases to lymph node Nos. 106recR and 106recL were, respectively, 9.1 and $0 \%$ in cStage I, 0 and $13.3 \%$ in cStage II, and 7.1 and 14.3 in cStage III, which were consistent with the previous reports $(4,6)$. More importantly, there were no significant differences in the prognoses of patients with surgical dissection of lymph nodes around RLN. Considering that RLN can be caused by RLN lymph node dissection, this procedure should be carefully considered in LtESCC patients; for example, this procedures should not be mandatory in cStage I/II for high risk patients such as elderly patients with low performance status or patients with impaired critical organs.

Limitations. The limitation of this research is that the number of cases is small. However, the survival rate of stage and tumor location in this study showed the same trend as other reports. For example, there is a report that the 5-year survival rate of each stage in surgical cases was 84.9/80.5/62.2/38.8/22.8/14.2\% in cStage 0/I/II/III/IVA/IVB, respectively (13). The 5-year survival rate of tumor location in surgical cases was 44.8/50.5/45.6\% in Ut/Mt/Lt, respectively, and there was no significant difference (14). Therefore, survival rates of stage and tumor location in our research are thought to be consistent outcomes despite the small number of cases. There are also other limitations on lymph node dissection range in this current study due to a retrospective study. According to the current clinical guideline of the 7th UICC-TNM, the abdominal lymph node dissection extent is limited to Nos. 1, 2, 3, 7, 9 lymph nodes. Nos. 4, 8, and 11 lymph nodes are out of range of dissection, and extra-regional. Recurrences at such extra-regional regions were hardly seen except for particular cases, and then the lymph node metastasis rate would not increase even if all cases are dissected of lymph nodes noted in this study. Since No. 9 lymph node is dissected together with No. 7, it may be contained as No. 7 in our cases. It is not possible to discuss backwardly with No. 7 and No. 9 separately, and the accuracy of the rates were not definitive.

In conclusion, rates of metastases to abdominal lymph nodes were higher than those to thoracic and cervical lymph nodes in LtESCC. In the context of the potent preoperative chemotherapy and esophagectomy, lymph node dissection of cervical areas, para-aortic areas, and even putative RLN areas are not mandatory to all patients with LtESCC, while the best operation no doubt should include all such procedures in patients with no impaired conditions. Moreover, we should know existence of a rare ESCC patient with uniquely aggressive lymph node disease, which might not be indicated for esophagectomy. Biomarkers may select such particular patients in the future.

Finally, from our study, we will describe the rationale of lymph node dissection of LtESCC cancer. (1) Neck lymph node dissection is likely to be optional. As a reason, the pathological metastasis rate and recurrence rate of the cervical lymph nodes are extremely low. (2) Prophylactic dissection of the para-aortic lymph node is then considered overtreatment, because recurrences at the para-aortic area were infrequent, and we have alternate therapeutic option for cure-definitive CRT even after recurrence (12). (3) Allowing for the Kaplan-Meier curve, it could be possible to omit either of RLN dissection for elderly patients and organ dysfunction cases with cStage I/II according to the specific clinical situation.

\section{Acknowledgments}

Not applicable.

\section{Funding}

No funding was received.

\section{Availability of data and materials}

All data generated or analyzed during this study are included in this published article.

\section{Authors' contributions}

$\mathrm{HH}$ and KY designed the study and wrote the initial draft of the manuscript. $\mathrm{HH}$ and $\mathrm{KY}$ analysed and interpreted the data and assisted in the preparation of the manuscript. $\mathrm{KH}, \mathrm{HMo}, \mathrm{HMi}$, AE, MWas, YK and MWat contributed to the collection and interpretation of the data and critically reviewed the manuscript. All authors approved the final version of the manuscript and agree to be accountable for all aspects of the work in ensuring that questions related to the accuracy or integrity of any part of the work are appropriately investigated and resolved.

\section{Ethics approval and consent to participate}

Not applicable.

\section{Patient consent for publication}

Not applicable.

\section{Competing interests}

The authors declare that they have no competing interests.

\section{References}

1. Ozawa S, Tachimori Y, Baba H, Matsubara H, Muro K, Numasaki H, Oyama T, Shinoda M, Takeuchi H, Tanaka O, et al: Comprehesive registry of esophageal cancer in Japan, 2002. Esophagus 7: 7-22, 2010.

2. Li H, Zhang Y, Cai H and Xiang J: Pattern of lymph node metastases in patients with squamous cell carcinoma of the thoracic esophagus who underwent three-field lymphadenectomy. Eur Surg Res 39: 1-6, 2007.

3. Igaki H, Tachimori Y and Kato H: Improved survival for patients with upper and/or middle mediastinal lymph node metastasis of squamous cell carcinoma of the lower thoracic esophagus treated with 3-field dissection. Ann Surg 239: 483-490, 2004.

4. Fujita H, Sueyoshi S, Tanaka T and Shirouzu K: Three-field dissection for squamous cell carcinoma in the thoracic esophagus. Ann Thorac Cardiovasc Surg 8: 328-335, 2002. 
5. Shimada Y, Imamura M, Sato F, Maeda M, Kaganoi J, Hashimoto Y, Kan T, Nagatani S and Li Z: Indications for abdominal para-aortic lymph node dissection in patients with esophageal squamous cell carcinoma. Surgery 132: 93-99, 2002.

6. Ye K, Xu JH, Sun YF, Lin JA and Zheng ZG: Characteristics and clinical significance of lymph node metastases near the recurrent laryngeal nerve from thoracic esophageal carcinoma. Genet Mol Res 13: 6411-6419, 2014.

7. Katada N, Yamashita K, Katada C,Moriya H,Hosoda K, Mieno H, Higuchi K, Komori S, IshiyamaH,Hayakawa K, et al: Neoadjuvant chemotherapy using concurrent Docetaxel/CDDP/5-FU (DCF) in esophageal squamous cell carcinoma and its short-term prognosis. Esophagus 11: 173-181, 2014.

8. Yamashita K, Katada N, Moriya H, Hosoda K, Mieno H, Katada C, Koizumi W, Hoshi K and Watanabe M: Neoadjuvant chemotherapy of triplet regimens of docetaxel/cisplatin/5-FU (DCF NAC) may improve patient prognosis of cStage II/III esophageal squamous cell carcinoma-propensity score analysis. Gen Thorac Cardiovasc Surg 64: 209-215, 2016.

9. Japanese Society for Esophageal Disease: Guideline for the clinical and pathological studies on carcinoma of the esophagus. 10th edition, revised. Kanehara \& Co., Ltd., Tokyo, 2002.

10. Nishimaki T, Suzuki T, Tanaka Y, Nakagawa S, Aizawa K and Hatakeyama K: Evaluating the rational extent of dissection in radical esophagectomy for invasive carcinoma of the thoracic esophagus. Surg Today 27: 3-8, 1997.
11. Motoyama S, Kitamura M, Saito R, Maruyama K, Okuyama M and Ogawa J: Outcome and treatment strategy for mid- and lower-thoracic esophageal cancer recurring locally in the lymph nodes of the neck. World J Surg 30: 191-198, 2006.

12. Yamashita K, Hosoda K, Moriya H, Katada C, Sugawara M, Mieno H, Komori S, Katada N and Watanabe M: Prognostic advantage of docetaxel/cisplatin/ 5-fluorouracil neoadjuvant chemotherapy in clinical stage II/III esophageal squamous cell carcinoma due to excellent control of preoperative disease and postoperative lymph node recurrence. Oncology 92: 221-228, 2017.

13. Tachimori Y, Ozawa S, Numasaki H, Ishihara R, Matsubara H, Muro K, Oyama T, Toh Y, Udagawa H and Uno T; Registration Committee for Esophageal Cancer of the Japan Esophageal Society: Comprehensive registry of esophageal cancer in Japan, 2010. Esophagus 14: 189-214, 2017.

14. Yang HX, Hou X, Liu QW, Zhang LJ, Liu JG, Lin P and Fu JH: Tumor location does not impact long-term survival in patients with operable thoracic esophageal squamous cell carcinoma in China. Ann Thorac Surg 93: 1861-1866, 2012. 